\title{
Sistemas automatizados para el control del recurso hídrico y variables ambientales bajo invernadero: aplicaciones y tendencias ${ }^{1}$
}

\section{Automated systems for water resources and environmental parameters control in greenhouses: applications and tendencies}

\author{
H. T. Tangarife, S. X. Toro, y C. V. Carmona \\ Recibido: febrero 28 de 2020 - Aceptado: junio 25 de 2020
}

\begin{abstract}
Resumen- Esta revisión se propone identificar las aplicaciones más recientes relacionadas con la automatización bajo invernadero con enfoque al uso adecuado del recurso hídrico y control de variables ambientales. La revisión hace parte del proyecto de investigación aplicada, diseño e implementación de un sistema SCADA para control del recurso hídrico y variables ambientales asociadas bajo invernadero mediante PLC. Se realiza una búsqueda detallada en revistas indexadas, nacionales e internacionales, páginas web, base de datos, y empresas relacionada al sector y se clasifica la información acorde a algunos criterios diferenciados; supervisión de variables bajo invernadero en sistemas irrigados, control automático del recurso hídrico y variables ambientales, adquisición de información para irrigación bajo invernadero, tendencias de la automatización en sistemas irrigados. A partir de la información se identifican las tecnologías recientes algunas aplicaciones, las tendencias y la necesidad de aplicarlas en el sector agrícola; como aporte para reducir el impacto de la migración de la mano de obra del campo a la ciudad.
\end{abstract}

Palabras clave- Automatización agrícola, control, invernadero, recurso hídrico, tecnología agrícola, variables ambientales.

1 Producto derivado del proyecto de investigación "Diseño e implementación de un sistema SCADA mediante PLC para manejo de riego y variables ambientales asociadas bajo invernadero". Presentado por el Grupo de Investigación GICEMET y Presentado por el Grupo de Investigación CIBA, del Servicio Nacional de aprendizaje - SENA.

H. I. Tangarife Sennova, Servicio Nacional de aprendizaje - SENA. Bogotá, Colombia, email: hitangarife@misena.edu.co.

S. X. Toro. Líder Sennova, Servicio Nacional de aprendizaje - SENA. Bogotá, Colombia, email: storom@sena.edu.co.

C. V. Carmona. Universidad Nacional de Medellín. Medellín, Colombia, Email: cvcarmona@unal.edu.co.

Como citar este artículo: Tangarife, H. I., Toro, S. X., y Carmona, C. V. Sistemas automatizados para el control del recurso hídrico y variables ambientales bajo invernadero: aplicaciones y tendencias, Entre Ciencia e Ingeniería, vol. 14, no. 27, pp.91-98, enero-junio 2020.

DOI: https://doi.org/10.31908/19098367.1796.

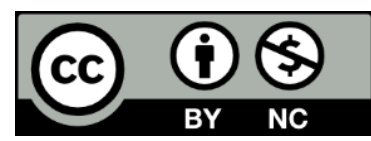

Attribution-NonCommercial 4.0 Intenational (CC By-NC 4.0)
Abstract - This review paper makes part of the applied research project "Design and implementation of a SCADA system to control the water resources and associated environmental parameters through PLC in greenhouses" ascribed to the research group GICEMET in the National Learning Service-SENA-. This study aims at identifying the applications of the agricultural automation to include models of supervision, control and information acquisition in the water resources and environmental parameters management for crops cultivated in greenhouses. A comprehensive search was carried out in national and international indexed journals, webpages and databases. The information was categorized based on several differentiated criteria: supervision under greenhouses, automatic control of water resources and environmental parameters, information acquisition for irrigation under greenhouses, and technological tendencies of the automation in the field. This review allowed to conclude about the advantages of the implementation of this kind of automation compared to conventional agricultural systems, as well as, poses the necessity to apply it in the agricultural sector as a contribution to reduce the impact of the workforce migration from the rural areas to the urban areas.

Keywords - Agricultural automation, agricultural technology, control, environmental variables, greenhouse, hidric resource.

\section{INTRODUCCIÓN}

GL agua no es un recurso inagotable; siendo un elemento fundamental para la vida, es también importante en los procesos sociales, productivos, en la generación de energía. Sin embargo, durante décadas se ha hecho un uso inadecuado en casi todas las actividades humanas. A esto se suma que, asociado al crecimiento de la población, la demanda y consumo del líquido aumenta. Hoy cuando se está agotando y su calidad deteriorando, no se vislumbran modelos económicos que busquen fortalecer el agro, no solamente desde el ámbito de la seguridad alimentaria, sino de necesidad de implementación de un desarrollo sostenible, que obliga al cuidado del ambiente, fortaleciendo otros sectores de la economía.

Por ello, y conscientes de la necesidad de mirar con compromiso la implementación de un desarrollo sostenible, 
procurando el cuidado del medio ambiente, sin descuidar la seguridad alimentaria; se abre una gran puerta a la preservación de las fuentes hídricas como parte importante del proceso con la implementación del sistemas de supervisión, control y adquisición de datos - SCADA, que además aporta innovación para el cuidado del medio ambiente; facilita la planificación del uso del recurso hídrico, y permite realizar control de plagas y enfermedades.

El cultivo bajo invernadero siempre ha permitido obtener vegetación de calidad y mayores rendimientos en cualquier momento del año, logrando mantener los cultivos en épocas de baja productividad. Un invernadero facilita el mantenimiento de parámetros físicos, como son temperatura del aire y del suelo, humedad relativa, porcentaje de $\mathrm{CO} 2$, iluminación, etc., en las condiciones óptimas para el desarrollo de las plantas en el interior o al menos en unas condiciones óptimas respecto al ambiente exterior [1].

La implementación de invernaderos tecnificados con sistema de riego controlado a través de un sistema de monitoreo, adquiere importancia dentro del proceso de producción agrícola, pero aún hoy en la mayoría de las empresas y en general de los agricultores, ejercen control y monitoreo de forma manual; riego con mangueras, uso de reservorios con bombas de distribución del agua; midiendo su desarrollo por medio de modelos matemáticos o de acuerdo al ciclo de vida de las plantas, desarrollando sistemas de supervisión de tareas en las diferentes áreas, con las respectivas dificultades que ello representa, dadas por la necesidad de hacer mediciones o monitoreo de las plantas. Es por ello que se hace necesaria la máxima tecnificación de los procesos de control y monitoreo de los diferentes cultivos, controlando en tiempo real condiciones claves como temperatura y humedad, de modo que se logren mejores resultados y a menor costo.

Un primer paso para sustentar la importancia del control de variables del microclima bajo invernadero se fundó en una exhaustiva revisión bibliográfica que permitió optar por la implementación de una tecnología que permite monitorear de forma remota, y controlar las variables de temperatura y humedad, adquirir y almacenar datos, así como presentar esta información en forma gráfica, en tiempo real, permitiendo conocer la trazabilidad.

Inicialmente se revisa a la supervisión de variables y se los diferentes modelos utilizados; en un segundo paso se revisan y analizan diferentes sistemas de control del microclima bajo invernadero a través del riego, luego se analizan los modelos de adquisición de información y finamente se determinan las tendencias de la automatización en el sector agrícola.

\section{SUPERVISIÓN DE VARIABLES BAJO INVERNADERO EN SISTEMAS IRRIGADOS}

El incremento de la población y por tanto de la demanda de alimentos, ha generado que la agricultura se enfrente a retos importantes. Estos retos, se encuentran enmarcados en fortalecer la estrategia de cultivos sostenibles y perdurables en el tiempo. Para este caso, los sistemas bajo invernadero cumplen este propósito, ya que son construcciones que permiten durante cualquier época del año obtener una producción continua, y además de contar con sistemas de irrigación, para reducir el gasto de agua, los costos y mejorar la calidad en la producción, por tanto, es necesario controlar y monitorear en todo momento variables como; humedad relativa, temperatura [2], luz, para garantizar que en todo el ciclo, el cultivo se encuentre en las condiciones ambientales óptimas.

Para lograr que un sistema bajo invernadero cumpla con el propósito para el cual fue diseñado, es necesario garantizar que el microclima cumpla con las condiciones adecuadas según el cultivo. Para ello, es necesario contar con un sistema completo de supervisión que le indique al usuario final las condiciones en las que se encuentra el sistema. actualmente, la supervisión de los sistemas bajo invernadero, se encuentran relacionados a cuatro temas importantes [3]: I) modelos climáticos en invernaderos [4]-[6], II) la red de sensores inalámbricos (WSN) [7]-[9], III) monitoreo remoto y sistemas de supervisión y adquisición de datos (SCADA) [2], [10] y IV) procesamiento de imágenes [11]. A continuación, se presentará información de cada uno de estos temas.

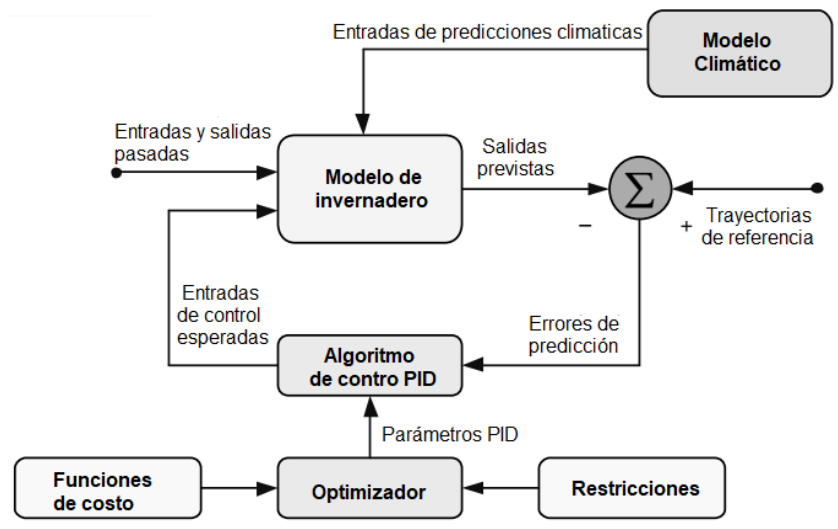

Fig. 1. Modelo del invernadero para una estructura del controlador PID adaptativo [4].

Para el caso I), con el desarrollo de los modelos climáticos en invernaderos, se busca un manejo y control más eficiente del sistema, ya que estos permiten predecir y simular este tipo de entornos. Proyectos como el de Boaventura presentan los comportamientos dinámicos de un invernadero, usando modelos lineales y no lineales [4], en donde se emplea la temperatura del aire, la humedad y la concentración del dióxido de carbono, para finalmente controlar el sistema con un PID adaptativo Fig. 1. Por otro lado se presenta el diseño de un modelo MPC (Model predictive control) basado en la optimización por enjambre de partículas (PSO) para la temperatura [6]. Con estos dos casos se puede evidenciar que existen avances de diferentes complejidades para dar solución al modelado dinámico de las variables de interés en sistemas bajo invernadero. Es importante destacar que el modelado dinámico no es el foco de este documento, pero es importante conocer las tendencias actuales relacionadas con modelos dinámicos bajo invernadero.

Para el caso II), la red de sensores inalámbricos o Wireless Sensor Network (WSN), es un modelo de transmisión de información que consiste en dispositivos autónomodistribuidos para monitorear variables físicas o ambientales (ver Fig. 2). Esta red, se compone de nodos de sensores, nodos receptores, una estación base, servidor y terminales de 
usuario. El nodo de sensores se encarga del monitoreo in-situ; el nodo receptor, se encarga de la transmisión de datos capturados, usando comunicación inalámbrica (ZigBee, Bluetooth, Wibree and WiFi) usualmente P2P; la estación base, busca que la información recolectada de los nodos receptores sea enviada a la estación base a través de una red GPRS; el servidor, recibe los datos desde la estación base y la terminal de usuario, se conecta al servidor a través de internet [8]. El uso de estos modelos en el contexto de la agricultura como lo menciona [8] se encuentra en un estado avanzado, además en este mismo documento se reporta que para sistemas irrigados usando WSN para el monitoreo de las variables en invernaderos se logra obtener entre un $30 \%$ a un $60 \%$ en la conservación del agua. Información que no solo se reporta en [8], sino también en otros artículo que enfatizan en la eficiencia de este modelo de sistema de comunicación para sistemas irrigados [9], [12]-[14].

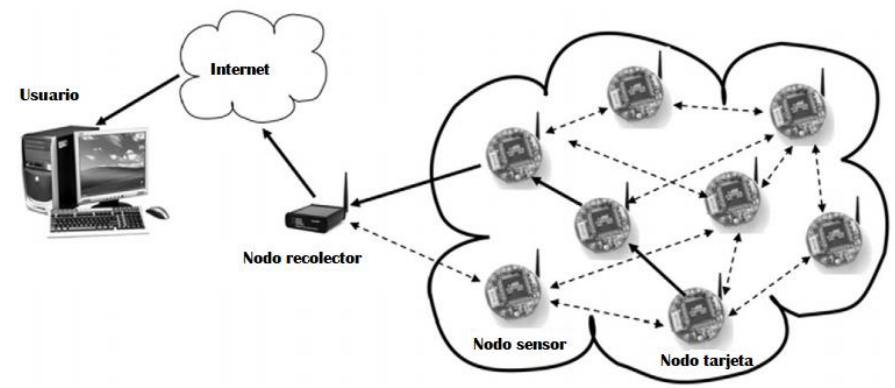

Fig. 2. Red de sensores inalámbricos o Wireless Sensor Network (WSN) [8].

Para el caso III), el monitoreo remoto y sistemas de supervisión y adquisición de datos (SCADA), es importante destacar, para este caso en particular, como la industria 4.0 (convergencia de las tecnologías de la información y la comunicación), generan nuevas innovaciones tecnológicas en el área de la agricultura [10]. Ya que por su versatilidad es posible conocer toda la cadena productiva del proceso, desde las necesidades del usuario que permiten tener cultivos personalizados que satisfagan al usuario final. Esto conlleva a la optimización de la oferta y demanda de forma automática. Uno de los ejemplos de esto, es el uso del internet da las cosas (IoT) para supervisar los cultivos bajo invernadero [15]. Yu presenta un modelo de supervisión donde se monitorea y diagnostica el estado del cultivo, además de la operación del invernadero de forma remota [16]. Por otro lado Karim presenta un sistema basado en IoT para la gestión del riego del cultivo [17], usando una interfaz gráfica donde se puede monitorear en tiempo real las variaciones de las condiciones del suelo.

Por último, otro de los sistemas relacionados con la supervisión de variables bajo invernadero es el procesamiento de imágenes. El procesamiento de imágenes, se puede dividir en tres categorías [18]: procesos de bajo, medio y alto nivel. El bajo nivel se relaciona a mejora de contraste, ruido y nitidez de la imagen; el nivel medio implica tareas tales como segmentación, la descripción y la clasificación (reconocimiento) de objetos individuales y el alto nivel implica "dar sentido" a un conjunto de objetos reconocidos, como en el análisis de imágenes, y en el otro extremo, realizar las funciones cognitivas normalmente asociadas con la visión. En este sentido, [11] proponen el uso de la visión artificial como una herramienta para la extracción de características, tales como parámetros de color (ver Fig. 3) y dimensión, textura, componentes químicos, detección de enfermedades, frescura para mejorar los sistemas agrícolas haciéndolos más eficientes, prácticos y confiables.

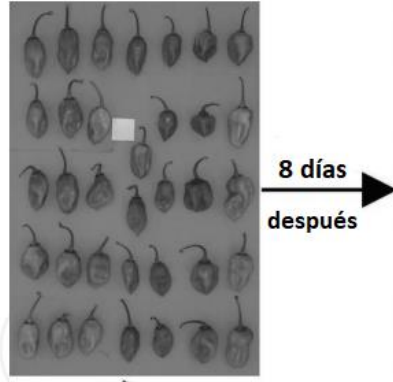

a)

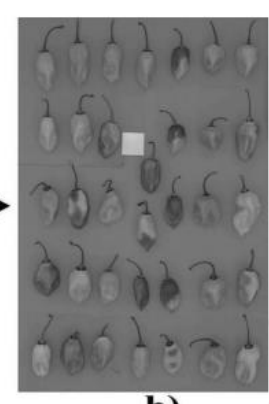

b)
Fig. 3. Captura de imágenes para análisis de color para el Chile. a) foto tomada 1 día después de recolección, b) foto tomada 8 días después [11].

Cada uno de los modelos de supervisión de variables bajo invernadero, presentan ventajas y retos significativos en su desarrollo, implementación y puesta a punto del sistema. Pero es importante destacar que según las necesidades particulares del sistema a implementar una u otra estrategia de supervisión puede brindar mejores elementos que permitan obtener un mejor desempeño del sistema.

\section{CONTROL AUTOMÁTICO DEL RECURSO HÍDRICO Y VARIABLES AMBIENTALES EN AGRICULTURA BAJO CUBIERTA}

El agua es un recurso limitado por las condiciones particulares de un área, adquiere gran importancia actualmente en los sistemas productivos debido a que el $69 \%$ agua dulce se consume en la agricultura [19], la mitad o más hace parte de procesos de incorporación a las plantas, transpiración y evaporación en cultivos, la otra parte se infiltra, fluye superficialmente hacia reservorios o hace parte de evaporación no productiva [20]. Es así como, el diseño e implementación de invernaderos aporta en la optimización de un ambiente adecuado y permite controlar todos los factores exógenos a las plantas. Muchos países en los cuales las condiciones de disponibilidad de los recursos son críticos, se han adelantado tecnológicamente a partir de la implementación de dispositivos que permiten realizar un manejo adecuado y riguroso del recurso.

Casos puntuales se presentan en países como: España en donde se desarrollan proyectos con la aplicación de diferentes casos de riego, se involucra un control basado en eventos, es decir, la ocurrencia de un evento es el que decide cuándo ejecutar la acción, en lugar del tiempo [21]. La Fig. 4 muestra la infraestructura, el modelo determina el volumen de agua [22]. Se maneja humedad ambiental, del suelo, temperatura variable que afectan los cultivos de interés agrícola.

En Chile se realizan estudios asociados de gran impacto en sistemas de producción hidropónica bajo invernadero en tomate, se plantea un sistema de suministro de agua y nutrientes a través cintas de riego, el objetivo es mantener la humedad y temperatura mediante la aplicación de agua y disponer de sensores de $\mathrm{pH}$ y conductividad eléctrica para aplicación de nutrientes, la Fig. 5 muestra la distribución del sistema. Se dispone de un reservorio de agua, alimentado 
mediante una motobomba, también se tiene un tanque con solución nutritiva [23]. Al igual que la hidroponía la aeroponía no cultiva en suelo y en reemplazo, usa soluciones nutritivas, en sistemas aeropónicos, para producción de papa, se realiza control de temperatura, humedad y nutrientes [24]. También se presentan aplicaciones en invernaderos inteligentes sobre cultivos en suelo donde se controlan variables, humedad, calefacción, ventilación [25].

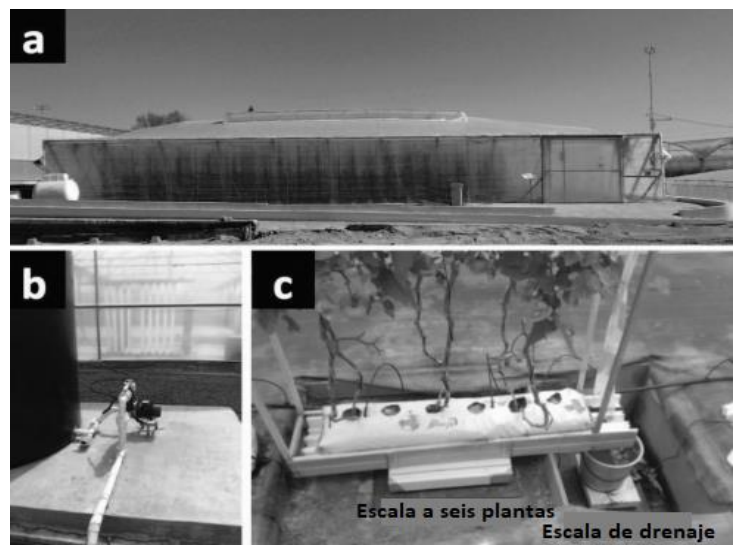

Fig. 4. Infraestructura para implementación del modelo de control [22].

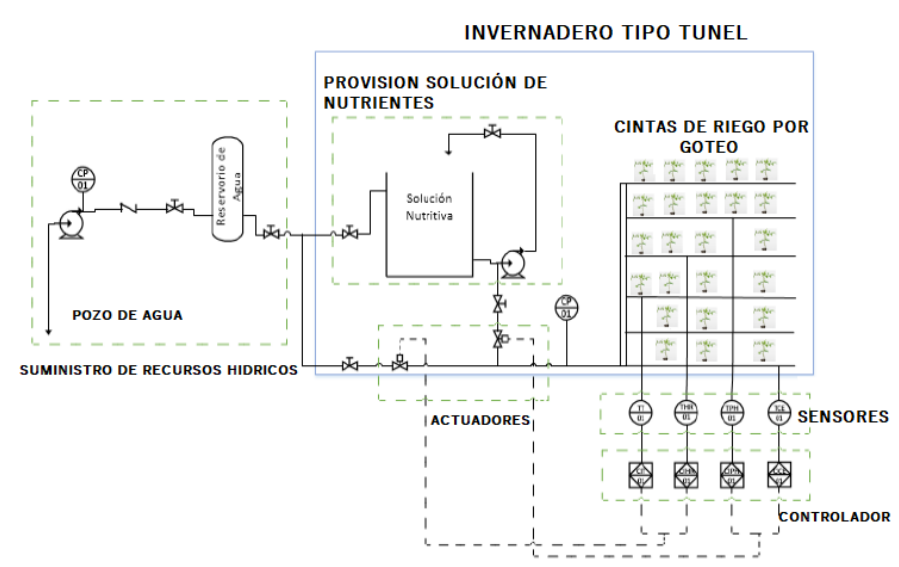

Fig. 5. Sistema de control para producción de tomate en cultivo hidropónico bajo invernadero [23].

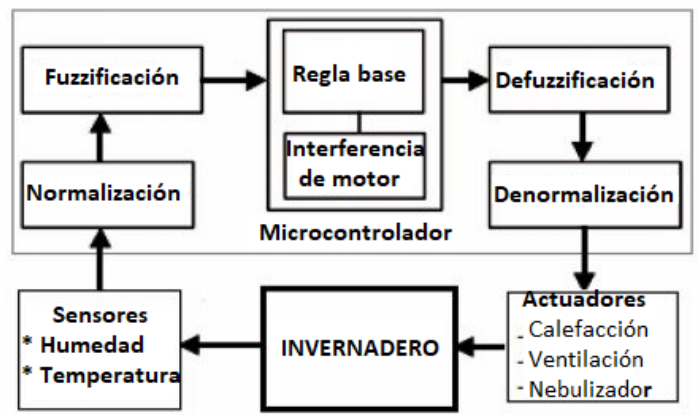

Fig. 6. Disposición del controlador fuzzy [28].

Se presenta la posibilidad de realizar control a distancia de humedad en invernadero mediante el uso de smartphone y a través de comunicación inalámbrica, el sistema realiza la lectura de la humedad en varios suelos y cultivos, mediante un control ON-OFF realiza la acción [26]. En Romania se usa energías alternativas para control de temperatura [27]. Por otro lado, el control inteligente con sensores vía inalámbrica es desarrollado por Kasim [28], que se propone realizar monitoreo y control ON-OFF en tiempo real mediante una red de sensores inalámbricos WSN, el microclima se controla con un conjunto de regla difusas Fig. 6.

Tradicionalmente se ha implementado el controlador ONOFF, sin embargo, otros avances relacionan modelos de control más rigurosos que involucran el riego como factor para control de la temperatura. Las imprecisiones, demandan implementar estudios en controladores tipo Proporcional, Integral, Derivativo PID, como solución más precisa y exacta. Investigadores realizan estudios comparativos de variables climáticas para controlar la temperatura y humedad, [29]. En la universidad de ciencia y tecnología de China se investiga alrededor de la mejora de los algoritmos de PID tradicional y de punto de ajuste mediante la implementación de un modelo de control predictivo, el Control Predictivo Generalizado GPC, es una de las estrategias que reúne muchas de las características los MPC's [30], [31].

Los invernaderos son sistemas de control de grandes inercias, y no linealidades, los modelos de manejo automatizados generan algoritmos de identificación de parámetros para lograr un control efectivo de la temperatura. Mediante simulación se muestran que GPC tiene un mejor rendimiento que el control PID tradicional, [32]. Otros autores plantean un modelo de control jerárquico multi-objetivo en donde se proponen mostrar una aproximación al control óptimo de crecimiento de cultivos en invernadero mediante el uso de técnicas de optimización para la maximización de beneficios y eficiencia en el uso del agua. [33].

Un control fuzzy PID es diseñado en china para manejo de variables en invernadero como temperatura ambiental mediante una válvula de agua caliente y fría, es decir, para aplicación de recurso hídrico [34]. Por otro lado, en Costa Rica, se realiza un Modelo Analítico de Lógica Difusa FLM para determinar "disponibilidad de agua", en donde, este término se cuantifica [35]. Los sistemas de calefacción de invernadero son multi-variables y de gran inercia, para solucionar el problema se utiliza la fuzzuficación de parámetros basado en el conocimiento experto [34]. Otros casos usan el conocimiento del agricultor asociado a contenidos de agua para fuzzificar el drenaje, lo que permite no acumular substrato, ni nutrientes. [35]. Sistemas fuzzy, son usados para la toma de decisiones, el modelo parte de una base de datos, con lluvia aleatoria [36]. En España se generan proyectos relacionados al uso eficiente del agua con sistema de apoyo en la toma de decisiones [37].

Existen otros tipos de control para la temperatura, variable muy dinámica bajo invernadero, con grandes retardos, no lineal y susceptible a perturbaciones, el modelo híbrido, analiza sistemas con dinámicas continuas como factores ambientales, y discretas como el estado de conmutación del equipo ambiental Fig. 7, [38].

La agricultura autónoma en el hogar, es decir, el jardín, antejardín, la terraza de las casas en contextos poblados, pueden ser usados para la producción agrícola. Sin embargo, debido a las ocupaciones y falta de tiempo para estar al tanto de la producción, se proponen modelos automatizados. En Malasia realizan avances en sistemas de control autónomo en invernaderos para agricultura doméstica, el proyecto plantea la 
importancia de la toma de decisiones en relación al riego asociado a las condiciones climáticas [39] Fig. 8, se estudia la posibilidad de irrigar a partir de la información que brindan sensores, temperatura humedad y piranómetro en donde se toma la señal, se proporciona al algoritmo de control para determinar el tiempo de riego [40].

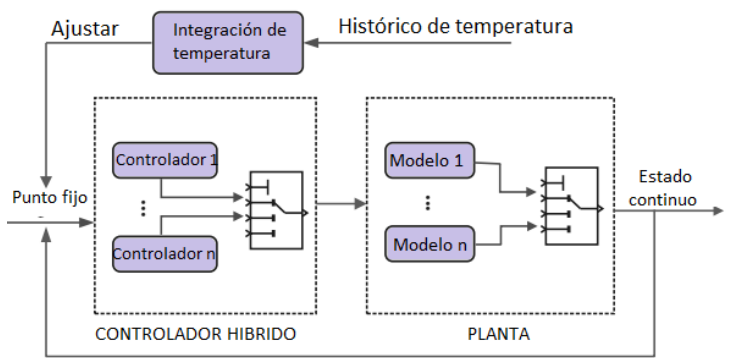

Fig 7. Diagrama de bloques del sistema de control [38].

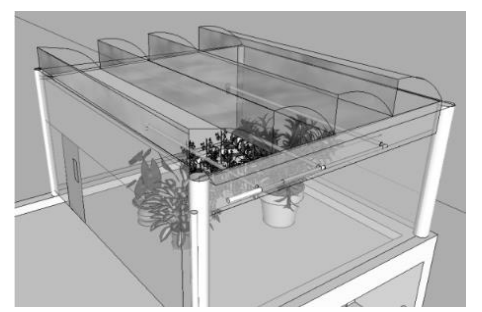

Fig. 8. Modelo de invernadero con ventilación en techo [39].

\section{ADQUISICIÓN DE INFORMACIÓN PARA IRRIGACIÓN BAJO INVERNADERO}

La falta de agua es el principal factor que puede generar estrés en las plantas, está relacionado con el agua absorbida por las raíces y la transpiración a través de las hojas, las condiciones hídricas de las plantas se pueden determinar a través de diferentes técnicas entre ellas cabe destacar; la emisión acústica (EA) [41], adquisición de información con sensores a través de IoT [42], detección de la demanda de luz de las plantas [43], entre otras.

En invernaderos modernos se realizan estudios asociados al manejo del riego mediante la implementación de sensores de emisión acústica mediante los cuales se adquiere señales en tiempo real de parámetros como: temperatura, humedad del aire, densidad de luz solar y $\mathrm{CO} 2$, el objetivo es irrigar automáticamente, se observa que la EA aumenta con el incremento de la transpiración, sin embargo, se identifica que el sistema es muy susceptible al ruido [41].

Internet de las cosas, IoT por sus siglas en inglés, ha sido una tecnología que ha facilitado la integración de internet con elementos tecnológicos en sistemas agrícolas, gana importancia por la facilidad que permite realizar la adquisición de datos y el control inteligente de los invernaderos. En Tianjin se diseña un sistema de recolección de información del suelo, las plantas y el ambiente a partir de una matriz de sensores, se registran datos de temperatura, humedad del suelo y del aire, CO2, intensidad de luz y se controlan bombas, ventiladores y otros equipos [42], proyecto con el mismo enfoque basado en arduino. [44], realiza la activación el riego de manera automática acorde a necesidades del sistema agrícola, mediante IoT se monitorea la temperatura, humedad y luz [45].

La luz es importante en el metabolismo de las plantas, la intensidad y calidad son factores clave en los procesos fotosintéticos, por tanto, los estudios de demanda de luz, temperatura y humedad en planta se hacen importantes en crecimiento. Estudios demuestran ser útiles para establecer las demandas de iluminación, [43], se pretende hacer que la iluminación permanezca constante para control de variables ambientales de impacto en la producción agrícola. En China se realizan proyectos para control y monitoreo de temperatura [46], se diseña un modelo con un dispositivo esclavo encargado recolectar los datos, toma la decisión de activar un extractor, se guardan la información y se grafica [47].

Con el desarrollo de controladores de bajo costo y el acceso libre a servicios de la web o aplicaciones, también se han implementado soluciones tecnológicamente accesibles a personas del común, el monitoreo mediante plataformas arduino que involucran IoT han facilitado los procesos de monitoreo y control ambiental bajo invernadero. Vimal menciona el caso de estudio para control de variables cuyo objetivo es alcanzar el crecimiento óptimo de las plantas, mediante mensajes de texto se visualizan parámetros ambientales que se envían al usuario, mediante una aplicación móvil [44], estudios similares de control [48] y monitoreo basado en android se describen en [49].

En otros casos no se usan tarjetas de desarrollo, sino el microcontrolador básico, la función es la misma obtener información de variables, visualizar, activar alarmas, controlar actuadores [50], la Fig. 9 Muestra un gráfico de distribución, también se realiza sensado de humedad y temperatura los paquetes se reciben en PC [51]. Proyectos basados en ZigBee plantean mejoras en la producción mediante el monitoreo, en donde se incluyen microcontroladores, sensores de humedad, temperatura, intensidad de luz y módulos de comunicación inalámbrica para adquisición de información [52].

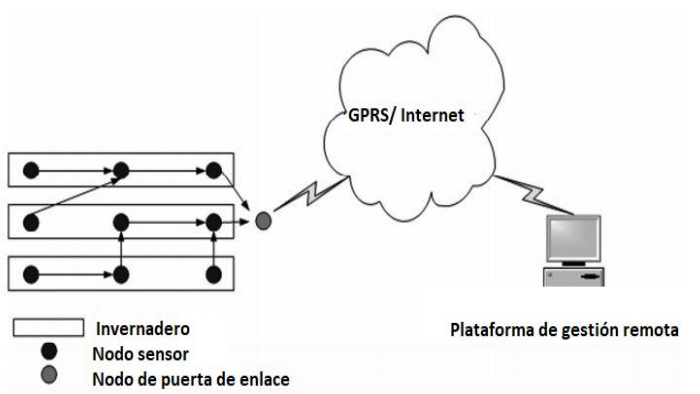

Fig 9. Estructura del sistema de adquisición de datos [51].

\section{TENDENCIAS DE LA AUTOMATIZACIÓN EN SISTEMAS IRRIGADOS}

Actualmente la disponibilidad de agua dulce se ve reducida por múltiples factores; contaminación, uso excesivo, pérdida por evaporación, entre otros, adicionalmente los ciclos del agua se ven afectados por cambios del clima que generan disminución significativa. La escasez del agua y la necesidad de cubrir la demanda de alimento hace que los sistemas agrícolas mejoren la producción lo que implica acelerados procesos de optimización. Con las nuevas tecnologías 
especialmente la automatización se transfiere mejoras al sector agrícola para amortiguar los gastos excesivos en recursos y los costos altos de producción que bajan significativamente los rendimientos.

Los modelos que controlan las variables ambientales y el recurso hídrico se presentan de múltiples formas tanto convencionales, como modelos más rigurosos y estables [53], es decir, se evalúa sistemas de control PID [54], control moderno, control inteligente [55], entre otros, con la idea de proporcionar estabilidad en las variables de interés agrícola, la tendencia en la actualidad es a mejorar los procesos de control, partiendo de que un invernadero es un sistema no lineal multivariable [56], que requiere aplicar un manejo integral para reducir el impacto de los factores externos mantener las condiciones estables para reducir el riesgo de enfermedades, plagas, malezas y mejorar los rendimientos.

Actualmente se realiza la detección de variables tanto ambientales como del suelo, en el primer caso, cabe destacar algunas como; la temperatura, humedad ambiental, intensidad de luz. La información proporcionada es útil al tomar una decisión, sin embargo, estas variables independientes del comportamiento del suelo, no entregan suficiente confiabilidad, dado esto, se extrae información como; humedad del suelo, conductividad, contenido de nutrientes, necesidad de riego, entre otras, que complementan la decisión de tratamiento químico o irrigación. Debido a que una sola variable no entrega información de confianza, puede llevar a tomar una decisión errada, sin embargo, la correlación de múltiples variables si la proporcionan. Siendo así, la información localizada adquiere importancia, ya que permite determinar el comportamiento de las variables ambientales y del suelo, entrega información relacionada en cada punto, la tendencia es a usar redes de sensores inalámbricos [57], nodos que permiten extraer información localizada a distancia y sin cableado [58], la información de cada punto contribuye con lo que se denomina agricultura de precisión donde se extrae información en áreas locales, es decir, lugares específicos, esta información se usa para determinar, necesidad de fertilización, riego, tiempos y cantidades de recursos que se deben suministrar al cultivo.

Una de las principales tendencias en la actualidad es a integrar las tecnologías. El uso de energías sostenibles como fuente de alimentación de los sistemas eléctricos hace parte de un avance significativo que aporta en la disminución de costos en el uso de las tecnologías. Igualmente, el monitoreo de variables ambientales se integra a internet de las cosas, IoT, en donde la toma de decisiones se fundamente en información de campo en tiempo real o en análisis asíncrono de la información almacenada en bases de datos obtenida a través del tiempo. El manejo en campo, es decir el control a distancia también tiende a integrarse a las tecnologías recientes con aplicaciones móviles, radiofrecuencia, mensajes de texto, IoT, entre otros.

El control de variables ambientales tiende a optimizarse en sistemas no lineales como invernaderos, mediante el uso de lógica difusa, aprendizaje automático, redes neuronales entre otros métodos, el pronóstico y la toma de decisiones mediante el aprendizaje se pueden implementar, adicionalmente los sistemas expertos pueden beneficiar la programación de los sistemas de riego para optimizar el uso del recurso hídrico y la adquisición automática de información mediante sistemas expertos.

\section{CONCLUSIONES}

El uso eficiente del recurso hídrico en la industria agrícola ha incentivado la implementación de nuevos sistemas de monitoreo y control en los procesos productivos. Para el caso particular tratado en este artículo, el monitoreo y control de sistemas bajo invernadero, incluye además del manejo eficiente del recurso hídrico, la necesidad constante de conocer el estado actual de procesos implementados en este tipo de sistemas de producción en ambientes controlados. Considerando esto, se evidencia en la revisión, que el monitoreo y control es de gran importancia ya que permite conocer el estado actual de las variables, tanto, del suelo como del ambiente o del entorno, donde esta información es pertinente para tomar acciones (automáticas o manuales) para el mejoramiento de la producción.

Se puede resaltar que, toda la información capturada no es suficiente con almacenarla o transmitirla a un entorno local, sino que, es necesario que este tipo de sistemas de monitoreo y control, se encuentren a la vanguardia de los avances tecnológicos mundiales. Por tal motivo, el procesamiento de imágenes, la industria 4.0, la big data, la inteligencia artificial, el internet de las IoT, y el uso de energías renovables como elementos generadores de energía al sistema, juegan un papel importante y diferenciador en el mejoramiento de los procesos productivos en este tipo de industria.

Cabe destacar que, para lograr avances significativos en la implementación eficiente de estos sistemas, es necesario continuar con la sinergia industria y sector educativo, ya que en este último es donde se desarrollan nuevas soluciones que permiten obtener resultados replicables y sistemáticos que ayudan mejorar los procesos productivos de este tipo de industrias.

La tecnología reciente puede generar avances significativos en la toma de decisiones para en el sector agrícola, muchas relacionadas al uso del recurso hídrico afectado por el cambio climático y otras a la mejora de los rendimientos que benefician directamente a la población en el aumento rápido, la automatización facilita las actividades agrícolas y se vuelve una herramienta útil en la trazabilidad de los productos y en la necesidad de decidir qué hacer ante determinadas circunstancias en el sector agrícola.

\section{AGRADECIMIENTOS}

Los autores extienden sus agradecimientos al Servicio Nacional de Aprendizaje SENA, especialmente a los subdirectores de los Centros de Biotecnología Agropecuaria CBA y Centro Metalmecánico CMM, y a los integrantes de los grupos de investigación GICEMET y CIBA respectivamente por el apoyo en los procesos.

\section{REFERENCIAS}

[1] D. Rozo, "Control y monitoreo de variables ambientales utilizando PLC y SCADA," Rev. Colomb. Tecnol. Av., vol. 2, no. 2, pp. 71-79, 2003.

[2] S. L. Li, Y. Han, G. Li, M. Zhang, L. Zhang, and Q. Ma, "Design and Implementation of Agricultral Greenhouse Environmental Monitoring 
System Based on Internet of Things," Appl. Mech. Mater., vol. 121-126, pp. 2624-2629, 2011.

[3] E. H. Gurban and G.-D. Andreescu, "Greenhouse environment monitoring and control: state of the art and current trends," Environ. Eng. \\& Manag. J., vol. 17, no. 2, pp. 399-416, 2018.

[4] C. J. Boaventura, "Greenhouse climate models: An overview," EFITA Conf., vol. 5, no. 9, pp. 823-829, 2003.

[5] N. Bennis, J. Duplaix, G. Enéa, M. Haloua, and H. Youlal, "Greenhouse climate modelling and robust control," Comput. Electron. Agric., vol. 61, no. 2, pp. 96-107, 2008.

[6] L. Chen, S. Du, Y. He, M. Liang, and D. Xu, "Robust model predictive control for greenhouse temperature based on particle swarm optimization," Inf. Process. Agric., vol. 5, no. 3, pp. 329-338, 2018.

[7] T. Ojha, S. Misra, and N. S. Raghuwanshi, "Wireless sensor networks for agriculture: The state-of-the-art in practice and future challenges," Comput. Electron. Agric., vol. 118, pp. 66-84, 2015.

[8] Aqeel-Ur-Rehman, A. Z. Abbasi, N. Islam, and Z. A. Shaikh, "A review of wireless sensors and networks' applications in agriculture," Comput. Stand. Interfaces, vol. 36, no. 2, pp. 263-270, 2014.

[9] K. P. Ferentinos, N. Katsoulas, A. Tzounis, T. Bartzanas, and C. Kittas, "Wireless sensor networks for greenhouse climate and plant condition assessment," Biosyst. Eng., vol. 153, pp. 70-81, 2017.

[10] J. Sung, "The Fourth Industrial Revolution and Precision Agriculture," in Automation in Agriculture, Securing Food Supplies for Future Generations, INTECH Open Access Publisher, 2018.

[11] E. Martínez Sandoval, J. R. Martínez Rosas, Miguel Enrique; Martínez Sandoval, and H. Miranda Velasco, Manuel Moises; Cervantes De Ávila, "Machine Vision Systems - A Tool for Automatic Color Analysis in Agriculture," in Automation in Agriculture, Securing Food Supplies for Future Generations, INTECH Open Access Publisher, 2018, pp. $125-148$.

[12] S. A. Nikolidakis, D. Kandris, D. D. Vergados, and C. Douligeris, "Energy efficient automated control of irrigation in agriculture by using wireless sensor networks," Comput. Electron. Agric., vol. 113, pp. 154163, 2015.

[13] D. L. Ndzi et al., "Wireless sensor network coverage measurement and planning in mixed crop farming," Comput. Electron. Agric., vol. 105, pp. 83-94, 2014.

[14] M. Srbinovska, C. Gavrovski, V. Dimcev, A. Krkoleva, and V. Borozan, "Environmental parameters monitoring in precision agriculture using wireless sensor networks," J. Clean. Prod., vol. 88, pp. 297-307, 2015.

[15] J. Gubbi, R. Buyya, S. Marusic, and M. Palaniswamia, "Internet of Things (IoT): A Vision, Architectural Elements, and Future Directions," Futur. Gener. Comput. Syst., vol. 29, no. 7, pp. 1645-1660, 2013.

[16] J. Yu and W. Zhang, "Study on Agricultural Condition Monitoring and Diagnosing of Integrated Platform Based on the Internet of Things," Comput. Comput. Technol. Agric. VI, vol. 392, pp. 244-250, 2013.

[17] F. Karim, F. Karim, and A. Frihida, "Monitoring system using web of things in precision agriculture," Procedia Comput. Sci., vol. 110, pp. 402-409, 2017.

[18] R. Gonzalez and R. Woods, Digital image processing, 3rd ed. New Jersey: Pearson Education, Inc., 2008.

[19] F. and agriculture organization FAO, "Uso del agua en la agricultura," 2005,2005 .

[20] F. Organización de las Naciones Unidas para la Alimentación, "Agua y cultivos, logrando el uso óptimo del agua en la agricultura," 2002. [Online]. http://www.fao.org/docrep/005/Y3918S/y3918s00.htm\#TopOfPage.

[21] S. Dormido, J. Sánchez, and E. Kofman, "Muestreo, control y comunicaci\{ó\}n basado en eventos," Rev. Iberoam. Autom\{áltica e Inform\{á\}tica Ind., vol. 5, no. 1, pp. 5-26, 2008.

[22] A. Pawlowski, J. A. Sanchez, J. L. Guzman, F. Rodriguez, M. Berenguel, and S. Dormido, "Event-based control for a greenhouse irrigation system," 2016 2nd Int. Conf. Event-Based Control. Commun. Signal Process. EBCCSP 2016 - Proc., 2016.

[23] V. Andaluz, A. Tovar, K. Bedón, J. Ortiz, and E. Pruna, "Automatic control of drip irrigation on hydroponic agriculture: Daniela tomato production," IEEE, vol. I, pp. 1-6, 2016.

[24] I. Idris and M. I. Sani, "Monitoring and control of aeroponic growing system for potato production," Proc. 2012 IEEE Conf. Control. Syst. Ind. Informatics, ICCSII 2012, pp. 120-125, 2012.

[25] S. Yan-Fang, C. Hebei Software Inst., Baoding, and S. J.-G.; X. YuQian, "Design and Application of Distributed Intelligent Greenhouse Computerized System," 2015 Seventh Int. Conf. Meas. Technol. Mechatronics Autom., pp. 331-334, 2015.
[26] A. N. Arvindan and D. Keerthika, "Experimental investigation of remote control via Android smart phone of arduino-based automated irrigation system using moisture sensor," 2016 3rd Int. Conf. Electr. Energy Syst. ICEES 2016, pp. 168-175, 2016.

[27] R. Grigoriu, A. Voda, N. Arghira, V. Calofir, and S. S. Iliescu, "Temperature Control of a Greenhouse Heated by Renewable Energy Sources," pp. 494-499, 2015.

[28] K. Al-Aubidy and M. Ali, "Real-time monitoring and intelligent control for greenhouses based on wireless sensor network," Multi-Conference ..., pp. 1-7, 2014.

[29] E. H. Gurban and G. Andreescu, "Comparison Study of PID Controller Tuning for Greenhouse Climate with Feedback-Feedforward Linearization and Decoupling," Int. Conf. Syst. Theory, Control Comput., pp. 1-6, 2012.

[30] A. Gómez, "Implementation of a Multivariable Predictive," pp. 195203,2009

[31] R. Jose, C. Coneo, and M. Sanjuan, "Diseño E Implementación De Un Controlador Predictivo Tipo," no. February, 2007.

[32] D. U. Y. Sen, M. A. Jiao, Q. I. N. L. Lin, Z. H. Bin, and W. U. Gang, "Application of Generalized Predictive Control Algorithm for Temperature Control in Modern Greenhouse," pp. 4342-4347, 2016.

[33] A. Ramírez, F. Rodríguez, J. L. Guzmán, and M. Berenguel, “A multiobjective approach to hierarchical control of greenhouse crop production," pp. 2519-2526, 2007.

[34] X. Jun and L. Zhou, "Design of Fuzzy PID Control Algorithm Facing to Greenhouse," 2017 9th Int. Conf. Intell. Human-Machine Syst. Cybern., pp. 130-133, 2017.

[35] A. Carrasquilla-Batista and A. Chacón-Rodríguez, "Proposal of a fuzzy logic controller for the improvement of irrigation scheduling decisionmaking in greenhouse horticulture," 2017 1st Conf. IEEE PhD Res. Microelectron. Electron. Lat. Am. PRIME-LA 2017, 2017.

[36] E. Giusti and S. Marsili-Libelli, "A Fuzzy Decision Support System for irrigation and water conservation in agriculture," Environ. Model. Softw., vol. 63, pp. 73-86, 2015.

[37] H. Navarro-Hellín, J. Martínez-del-Rincon, R. Domingo-Miguel, F. Soto-Valles, and R. Torres-Sánchez, "A decision support system for managing irrigation in agriculture," Comput. Electron. Agric., vol. 124, pp. 121-131, 2016.

[38] J. Ma, Y. Sen Du, L. L. Qin, G. Wu, and D. X. Wang, "Hybrid control of greenhouse temperature system based on crop temperature integration theory," Chinese Control Conf. CCC, pp. 2426-2431, 2017.

[39] M. A. Abas and M. Dahlui, "Development of greenhouse autonomous control system for Home Agriculture project," ICAMIMIA 2015 - Int. Conf. Adv. Mechatronics, Intell. Manuf. Ind. Autom. Proceeding conjunction with Ind. Mechatronics Autom. Exhib. IMAE, vol. 2015, no. Icamimia, pp. 12-17, 2016.

[40] M. Amir Abas, N. Amalia Sapiee, and M. Dahlui, "Autonomous Irrigation Hours through Loop Signals of Weather Sensors," Proc. AMS 2015 Asia Model. Symp. 2015 - Asia 9th Int. Conf. Math. Model. Comput. Simul., pp. 52-57, 2016.

[41] S. Yang, P. Lu, L. Okushima, and S. Sase, "Precision irrigation system based on detection of crop water stress with acoustic emission technique," Int. Conf. Inf. Acquis. 2004. Proceedings., pp. 444-447, 2004.

[42] T. Guo and W. Zhong, "Design and implementation of the span greenhouse agriculture Internet of Things system," Proc. 2015 Int. Conf. Fluid Power Mechatronics, FPM 2015, pp. 398-401, 2015.

[43] Y. Li, P. Niu, and Z. Su, "Design of greenhouse monitoring and control system based on LED lighting," 2015 12th China Int. Forum Solid State Light. SSLCHINA 2015, pp. 123-126, 2015.

[44] P. V. Vimal and K. S. Shivaprakasha, "IOT based greenhouse environment monitoring and controlling system using Arduino platform," 2017 Int. Conf. Intell. Comput. Instrum. Control Technol. ICICICT 2017, vol. 2018-Janua, pp. 1514-1519, 2018.

[45] Z. Li, J. Wang, R. Higgs, L. Zhou, and W. Yuan, "Design of an Intelligent Management System for Agricultural Greenhouses Based on the Internet of Things," Proc. - 2017 IEEE Int. Conf. Comput. Sci. Eng. IEEE/IFIP Int. Conf. Embed. Ubiquitous Comput. CSE EUC 2017, vol. 2, pp. 154-160, 2017

[46] S. Alyousif, N. F. Zainuddin, and B. Bin Hamzah, "Intelligent temperature control system at greenhouse," Int. J. Appl. Eng. Res., vol. 12, no. 9, pp. 1811-1814, 2017.

[47] S. Ma, "Design of intelligent monitoring system for greenhouse," Proc. 2010 Int. Conf. Multimed. Commun. Mediacom 2010, pp. 31-34, 2010.

[48] T. Li, G. Shi, J. Hou, M. Wei, X. Lang, and G. Zhang, "Greenhouse 
intelligent control system based on STM32F107 and switched Ethernet," J. Theor. Appl. Inf. Technol., vol. 46, no. 1, pp. 426-433, 2012.

[49] L. Zhang, C. Li, Y. Jia, and Z. Xiao, "Design of Greenhouse Environment Remote Monitoring System Based on Android Platform," Chem. Eng. Trans., vol. 46, pp. 739-744, 2015.

[50] Y. Jun, "Design of intelligent monitoring and controlling system for greenhouse," 2011 Int. Conf. Electr. Control Eng. ICECE 2011 - Proc., pp. 629-632, 2011.

[51] Q. NIU, "A desingn of modern green house environmental monitoring system," Asian Agric. Res., vol. 1, no. 1, pp. 57-60, 2017.

[52] W. Qui, L. Dong, F. Wang, and H. Yan, "Desing of intelligent greenhouse environment monitoring system based in zigbee and embedded technology," IEEE, pp. 12-13, 2014.

[53] M. A. K. Echaieb, F. T. E. Fabrizio, and A. I. A. Mami, "Fuzzy Decoupling Control of Greenhouse Climate," Springer J., pp. 28052812, 2015.

[54] H. Hu, L. Xu, and E. D. Goodman, "NSGA-II-based nonlinear PID controller tuning of greenhouse climate for reducing costs and improving performances," Springer J., pp. 927-936, 2014.

[55] M. Nadafzadeh and S. Abdanan, "Design and fabrication of an intelligent control system for determination of watering time for turfgrass plant using computer vision system and artificial neural network," Precis. Agric., no. 0123456789, 2018.

[56] A. Maher, E. Kamel, and F. Enrico, "An intelligent system for the climate control and energy savings in agricultural greenhouses," Energy Effic., pp. 1241-1255, 2016.

[57] J. A. Jiang et al., "A wireless sensor network-based monitoring system with dynamic convergecast tree algorithm for precision cultivation management in orchid greenhouses," Precis. Agric., vol. 17, no. 6, pp. 766-785, 2016.

[58] W.-M. Yang et al., "A Study on Greenhouse Automatic Control System Based on Wireless Sensor Network," Wirel. Pers. Commun., vol. 56, no. 1, pp. 117-130, 2009.

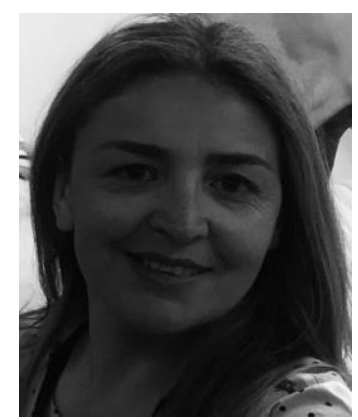

Sandra Ximena Toro Meléndez. Miembro del grupo de investigación CIBA CBA, Líder SENNOVA del Centro de Biotecnología Agropecuaria, Tecnóloga en Administración Agropecuaria del Centro de Biotecnología Agropecuaria, obtuvo su grado de Administradora de empresas agropecuarias en la Universidad Santo Tomás, en el año 2002 y en el año 2012 se graduó de Magíster en Sistemas Integrados de Gestión: Calidad, medio ambiente y riesgos laborales en la Universidad Internacional de La Rioja, España. Sus áreas de interés son el desarrollo de proyectos relacionados con la protección del medio ambiente, la seguridad y salud de los trabajadores y el sector agropecuario. ORCID: https://orcid.org/0000-0002-1052-1163.

Cindy Vanessa Carmona Cadavid. Obtuvo su grado en Ingeniería de Control en la Universidad Nacional de Colombia sede Medellín en 2013. Luego recibió su título de especialista en Robótica y Mecatrónica y Magíster en Ingeniería en la Universidad Pontificia Bolivariana, en 2017. Sus áreas de interés son el modelado de procesos para control, desarrollo de software y el uso de algoritmos evolutivos para la sintonía óptima de controladores, además del desarrollo de robótica para la exploración, monitoreo y conservación de entornos

ORCID: https://orcid.org/0000-0002-4967-6103 subacuáticos.

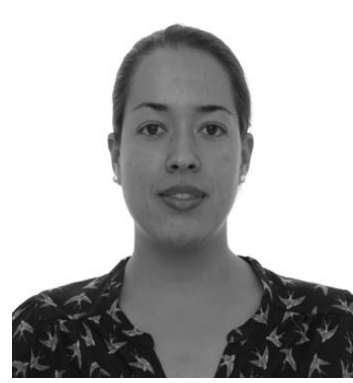

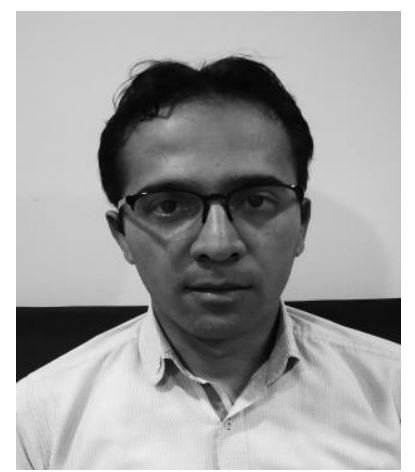

Héctor Iván Tangarife Escobar. Investigador del grupo de investigación GICEMET perteneciente al Centro Metalmecánico Servicio Nacional de Aprendizaje - SENA, área de mantenimiento industrial. Obtuvo su grado de ingeniero en Control de la Universidad Distrital Francisco José de Caldas Bogotá D.C, 2015. Luego realizó su especialización en informática y automática industrial de la misma universidad, en 2017, actualmente cursa maestría en instrumentación y automatización en la Universidad Antonio Nariño, Sede Bogotá D.C. Sus áreas de interés son la Automatización dirigida al sector agrícola, automatización industrial e instrumentación industrial ORCID: https://orcid.org/0000-0002-9416-385X. 